\title{
ON-GROUND ATTITUDE DETERMINATION IN THE HIPPARCOS MISSION
}

\author{
F. DONATI, E. CANUTO \\ Dipartimento di Automatica e Informatica, Politecnico di Torino, \\ Corso Duca degli Abruzzi 24, 10128 Torino, Italy \\ and \\ Centro di Studi sui Sistemi, Torino, Italy
}

\author{
J.L. FALIN, M. FROESCHLÈ, J. KOVALEVSKY \\ CERGA, Observatoire de la Côte d'Azur \\ 06130 Grasse, France
}

\begin{abstract}
An accurate on-ground reconstitution of the satellite three-axes attitude from the downlinked star mapper photon counts is an essential factor in the final accuracy of the Hipparcos Astrometric Catalogue. The reconstitution method developed by the FAST Consortium and currently on use to process Hipparcos data is the concern of this paper. After on outline of the main reconstitution modcls and algorithms, an accuracy assessment of the estimates, attitude and star coordinates, coming out from the first year data reduction, is presented; the assessment shows that attitude root mean square errors are already around the mission target of 0.1 arcseconds.
\end{abstract}

\section{Specifications of the three-axes attitude reconstitution.}

The satellite three-axes attitude is an essential data input to the first Hipparcos data reduction stage, the Reference Great Circle (RGC) reduction that processes the main grid star coordinates to estimate the programme stars RGC abscissae. The attitude is determined before the reduction of the main grid photon counts, by processing the star mapper photon counts and the a priori star coordinates available from the Input Catalogue or the best version of the Hipparcos Catalogue. The target attitude accuracy was derived from the overall error apportionment, leading to the requirement that each attitude component shall be a posteriori determinable with a root mean square error less than 0.1 arcseconds over a great circle.

In order to reach this accuracy with a good confidence, FAST Consortium planned to reconstitute the satellite attitude iteratively as far as the accuracy of the star coordinates shall have been improved by the data processing. Hence, two different attitude reconstitutions were studied and developed: the first year attitude reconstitution, based on the star coordinates available from the Input Catalogue; the standard attitude reconstitution, based on the first version of the Hipparcos Catalogue. A further improvement of the attitude, although limited to the longitudinal component, will be available form the great circle reduction; nevertheless, the improved attitude will be not fed back to Hipparcos data reduction; but it will constitute the basis of the secondary mission Tycho. This use explains the efforts to improve the attitude accuracy as far as possible, also beyond the Hipparcos mission requirements.

One of the main features of the FAST Consortium data reduction, is that attitude is reconstituted by processing only star mapper measurements, even if also on-board gyro readings are available. An analysis of the gyroscopes contribution to attitude accuracy, reported in [1], shew that their content of information shall have been negligible with respect to the one pro- 
vided by star mapper observations.

Attitude reconstitution requires two separate data processing, to be run sequentially:

1) the star mapper data reduction, providing the transit times of bright programme stars across the star mapper slits; transit time accuracy was set up to be less than 0.1 arcseconds to get attitude accuracy target with good confidence;

2) the attitude determination, processing the star mapper transit times and the star coordinates of the best available catalogue and providing the three attitude components of the satellite and, until the only Input Catalogue will be available, the RGC coordinates of the programme stars..

\section{The star mapper data reduction and its accuracy.}

The objective of the star mapper data reduction is to estimate from the photon counts of the same star passage, collected by the photometric channels $V$ and $B$ :

1) the star transit time $t$ across the vertical and the inclined reference lines, each one defined as the optical barycentre of the corresponding slit group (geometric estimate),

2) the star intensities $a_{B}$ and $a_{V}$ and the background intensities $b_{B}$ and $b_{V}$ in the two star mapper colours (photometric estimates).

Only geometric estimates are relevant to attitude reconstitution. Geometric and photometric estimates might be computed by fitting the expected Poisson-like distribution of the photon counts, collected during a single star transit, to the actual counts record, according to the maximum likelihood criterion. In practice this procedure could bring very often to transit lime errors very beyond the accuracy upper limit of 0.1 arcseconds, because of the contemporary passage of brighter parasitic stars or the observation of too faint programme stars. To eliminate such outliers, that severely affect attitude accuracy, maximum likelihood estimation is applied only to photon counts that are detected to belong to an expected bright and single programme star.

The accuracy of the transit time estimates has been assessed from the estimated residuals and an accurate estimate of the transit velocity. Table 1 shows typical values of the a posteriori estimates of the transit time errors, that appear to be very stable from one RGC to another; units have been transformed from time to angular ones.

Table 1. A posteriori transit times root mean square errors, in milli-arcseconds.

\begin{tabular}{|c|c|c|}
\hline Magnitude & Vertical slits & Inclined slits \\
\hline$<6$ & 6 & 8 \\
$6-7$ & 10 & 16 \\
$7-8$ & 18 & 27 \\
$8-9$ & 29 & 45 \\
$9-10$ & 46 & 67 \\
\hline
\end{tabular}

\section{Attitude determination, star coordinate updates and their accuracy.}

The objective is to determine, at a rate of about $0.5 \mathrm{~Hz}$, three angles defining the orientation of the Hipparcos telescope reference frame $(O, x, y, z)$ with respect to a celestial reference frame $\left(O, X_{1}, X_{2}, X_{3}\right)$ having the $X_{3}$ axis aligned with the pole of the Reference Great Circle and the $X_{l}$ axis aligned with the ascending node on the ecliptic plane. The three angles could be the clas- 
sical Eulerian angles $(\alpha, \beta, \delta)$ used in astronomy; instead, for modeling reasons related to the dynamics of the satellite, an hybrid triad of angles $(\psi, \theta, \phi)$ was chosen, being defined by:

$$
\psi=\alpha+\delta, \quad \theta=\beta, \phi=\alpha
$$

where $\psi$ is equivalent to the longitude of the perigee in Celestial Mechanics.

At time $t$, when a programme star crosses the reference line of a star mapper group of slits $f$, a constraint equation linking the parametric models of the attitude angles $\psi, \theta, \phi$, the catalogue coordinates $\zeta, \eta$, the star transit time estimate $t^{\prime}$ and the calibrated instrument parameters $\lambda$, can be written:

$$
h_{f}\left(\zeta, \eta ; \psi\left(t^{\prime}, \mu\right), \theta\left(t^{\prime}, \mu\right), \phi\left(t^{\prime}, \mu\right) ; \lambda\right)=\varepsilon_{f}
$$

where $\mu$ is the parameter vector of attitude models and $\varepsilon_{f}$ is a variable, taking into account the effect of all possible errors: catalogue, calibration, modeling and transit time errors.

Since an attitude reconstitution algorithm has to maximize the likelihood function of the measurement errors, it strictly depends on the statistical properties of the different error variables included in eq. 2. Two different error statistics have been defined and consequently two different estimation algorithms have been developed to take into account the different epochs of data reduction.

1) First year attitude reconstitution. The instrument calibration errors and the attitude modeling errors were assumed to be negligible; instead transit time errors and catalogue errors were taken into account and were modeled as uncorrelated random variables with known variance. At this stage the reconstitution algorithm gives at the same time the three attitude angles and the updates of the star coordinates in the RGC frame.

2) Standard attitude reconstitution. When a first Hipparcos catalogue will be available, the errors affecting the star coordinates shall be retained negligible with respect to transit time errors; the latter ones will remain the only cause of inaccuracy.

An explicit formulation of the measurement eq. 2 and of the algorithms may be found in [2]. The most critical problem was how to model the time evolution of the attitude components during all the mission. After theoretical studies and several tests on simulated and mission data two different attitude models appeared to be the most suitable:

1) Long term dynamical model. When the satellite is operating in absence of solar eclipses, perturbing torques are either constant or they show the period impressed by the satellite 2-hours rotation and dominant 3rd and 6th harmonics due to the hexagonal shape of the spacecraft. As a consequence any time realization of the attitude angles can be developed into a finite trigonometric expansion that remains almost invariable during the mission (attitude dynamical smoothing) and a polynomial expansion (secular part) depending on the gas jet actuations generated by the on-board attitude control.

2) Normal short term model. When the satellite dynamics during a rotation period is not stationary, what happens during a solar eclipse, or when star mapper observations are partly missing, preceding considerations do not apply and it is not possible to use dynamical smoothing. In this cases appropriate polynomial expansions were used.

Till to present days only the original version of the Input Catalogue has been used as reference catalogue for the attitude reconstitution. Since the root-mean-square of the Input Catalogue errors can be very roughly evaluated to be greater than 0.25 arcseconds, depending on the sky region and star magnitudes, they cannot be neglected in eq. 2 , leading to the use of the first year reconstitution algorithm, that offers the possibility of updating the star coordinates. In particular, 
after the attitude angles $\psi, \theta, \phi$ have been estimated, the star abscissa $\zeta$ is determined from vertical slits transits and, by knowing $\zeta$, one can estimate the ordinate $\eta$ from inclined ones. This reconstitution version has been up to now tested under a very large variety of cases to verify the statistical model of the measurement errors and to assess the attitude reconstitution accuracy.

The most significant tests were made by comparing the attitude and the star coordinates reconstituted from star mapper transit times and the ones obtained by smoothing the main grid coordinates projected on the reference great circle. Since the latter reduction reconstitutes only the longitudinal attitude and the RGC star abscissae, this method allows to derive a definite assessment only for the accuracy of the angle $\psi$ and the coordinate $\zeta$. The assessment is made by assuming that the attitude and star coordinate errors of the great circle solution are negligible if they are compared with the errors coming from the star mapper reduction. This assessment confirms the standard deviations estimated by the algorithm and based on the estimated star mapper errors and the a priori errors of the Input Catalogue.

Table 2 shows the estimated standard deviations of the attitude angles and the RGC star coordinates, obtained from first year data sets reconstituted with the dynamical smoothing. When dynamical smoothing cannot be applied, attitude accuracy is degraded; in this case figures below have to be multiplied by about 1.5 .

Table 2. Estimated standard deviations of attitude angles and star coordinates from first year reconstitution; unit: milli-arcseconds.

\begin{tabular}{|l|c|c|c|c|c|c|}
\hline Reconstitution & \multicolumn{2}{|c|}{ Longitudinal } & \multicolumn{3}{|c|}{ Transversal variables } \\
\hline Attitude & $\psi$ & $35-55$ & $\theta$ & $60-90$ & $\phi$ & $130-170$ \\
\hline Star Coordinates & $\zeta$ & $40-50$ & $\eta$ & $70-90$ \\
\hline
\end{tabular}

\section{Conclusions and acknowledgments}

The Hipparcos attitude unitil now reconstituted by FAST Consortium, from star mapper observations and the Input Catalogue star positions, shows very coherent results from one data set to another. The assessed accuracy, at least in the cases of dynamical smoothing, appears already below the target accuracy of 0.1 arcseconds. A further improvement of the accuracy is expected when the first Hipparcos Catalogue will be available.

The methodologies described in this paper and the relevant computer programs were developed at Centro di Studi sui Sistemi, Torino (Italy). The programs were then transferred to Centre National d'Etudes Spatiales, Toulouse (France), where all the data reduction is performed under the scientific responsibility of CERGA, Grasse (France).

\section{References}

1. Donati F. \& alii (1984), High-accuracy On-ground Attitude Reconstitution for the ESA Astrometry Mission Hipparcos, Proc. 9th World Congress of the Int. Fed. of Automatic Control, Budapest (Hungary), vol. III, 18-23.

2. Donati F., Canuto E., Fassino B. and Belforte P. (1986), High-accuracy On-ground Attitude Reconstitution for the ESA Astrometry Hipparcos Mission, Manuscripta Geodaetica, 11, 115123 